\title{
HS-1793, a resveratrol analogue, downregulates the expression of hypoxia-induced HIF-1 and VEGF and inhibits tumor growth of human breast cancer cells in a nude mouse xenograft model
}

\author{
DONG HWAN KIM ${ }^{1 *}$, BOKYUNG SUNG ${ }^{1 *}$, JIN-AH KIM $^{1}$, YONG JUNG KANG ${ }^{1}$, \\ SEONG YEON HWANG ${ }^{1}$, NA-LAM HWANG ${ }^{1}$, HONGSUK SUH $^{2}$, YUNG HYUN CHOI ${ }^{3}$, \\ EUNOK IM $^{1}$, HAE YOUNG CHUNG ${ }^{1}$ and NAM DEUK KIM ${ }^{1}$
}

\author{
Departments of ${ }^{1}$ Pharmacy, Molecular Inflammation Research Center for Aging Intervention, \\ and ${ }^{2}$ Chemistry and Chemistry Institute for Functional Materials, Pusan National University, Busan 46241; \\ ${ }^{3}$ Department of Biochemistry, Dongeui University College of Oriental Medicine, Busan 47340, Republic of Korea
}

Received October 7, 2016; Accepted June 16, 2017

DOI: 10.3892/ijo.2017.4058

\begin{abstract}
A synthetic analogue of resveratrol, 4-(6-hydroxy2-naphtyl)-1,3-benzenediol (HS-1793), with improved photosensitivity and stability profiles, has been recently reported to exert anticancer activity on various cancer cells. However, the molecular mechanism of action and in vivo efficacy of HS-1793 in breast cancer cells have not been fully investigated. In the present study, we evaluated the effect of HS-1793 on hypoxia-inducible factor- $1 \alpha$ (HIF-1 $\alpha)$, which drives angiogenesis and the growth of solid tumors, in addition to the in vivo therapeutic effects of HS-1793 on breast cancer cells. HS-1793 was found to inhibit hypoxia $(1.0 \%$ oxygen)-induced HIF-1 $\alpha$ expression at the protein level, and its inhibitory effect was more potent than that of resveratrol in MCF-7 and MDA-MB-231 breast cancer cells. Furthermore, HS-1793 reduced the secretion and mRNA expression of vascular endothelial growth factor (VEGF), a key mediator of HIF-1-driven angiogenesis, without affecting cell viability. To evaluate the anticancer effects of HS-1793 in vivo, triple-negative MDA-MB-231 breast cancer xenografts were established in nude mice. HS-1793 significantly suppressed the growth of breast cancer tumor xenografts, without any apparent toxicity. Additionally, decreases in Ki-67, a proliferation index marker, and CD31, a biomarker of microvessel density, were observed in the tumor tissue. Expression of HIF-1 and VEGF was also
\end{abstract}

Correspondence to: Professor Nam Deuk Kim, Department of Pharmacy, College of Pharmacy, Pusan National University, Busandaehag-ro, 63beon-gil 2, Geumjeong-gu, Busan 46241, Republic of Korea

E-mail: nadkim@pusan.ac.kr

${ }^{*}$ Contributed equally

Key words: HIF-1, VEGF, resveratrol analogue, xenograft, breast cancer downregulated in xenograft tumors treated with HS-1793. These in vivo results reinforce the improved anticancer activity of HS-1793 when compared with that of resveratrol. Overall, the present study suggests that the synthetic resveratrol analogue HS-1793 is a potent antitumor agent that inhibits tumor growth via the regulation of HIF-1, and demonstrates significant therapeutic potential for solid cancers.

\section{Introduction}

Breast cancer is a prevalent type of cancer and in 2012, it was found to be the leading cause of cancer-related mortality in women worldwide (1). Clinically, breast cancer can be divided into distinct subtypes based on the expression of estrogen receptor (ER), progesterone receptor (PR), and amplification of HER-2/Neu, that have prognostic and therapeutic implications (2). Triple-negative breast cancer (TNBC) which is defined by the lack of ER, PR and HER-2 expression, accounts for $\sim 15 \%$ of all breast carcinomas (3). In particular, patients with TNBC have a poor outcome compared to the other subtypes of breast cancer, with the 5-year survival rate being lower than $30 \%$ (4).

There have been significant advances in detection and chemotherapy, which provide the best prognosis for long-term survival and improve quality of life. However, $70 \%$ of patients with breast cancer are inoperable due to tumor outgrowth or bone metastasis (5), possibly as a result of induced hypoxia (6). The hypoxic regions are easily found in most solid tumors due to the severe structural abnormality of tumor microvessels (7). Hypoxia plays a role as a negative prognostic and predictive factor owing to its multiple contributions to chemoresistance, radioresistance, angiogenesis, vasculogenesis, invasiveness, metastasis, resistance to cell death, altered metabolism and genomic instability (8). Thus, it is not surprising that hypoxia is associated with reduced survival in patient with several cancers (9). It has been reported that the hypoxic condition can govern TNBC progression (10), promoting adaptation through genes within the major hallmarks of cancer (11). TNBC is the breast cancer subtype most frequently associated with hypoxia 


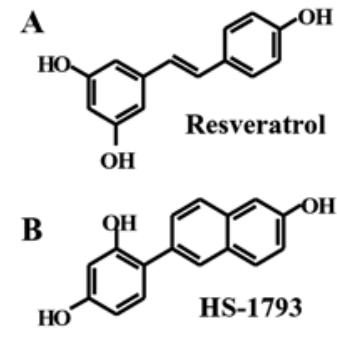

Figure 1. Chemical structures of resveratrol (3,4,5-trihydroxy-trans-stilbene) and HS-1793 [4-(6-hydroxy-2-naphthyl)-1,3-benzenediol].

and displays overexpression of hypoxia-inducible factor (HIF) target genes (12). Given the role in hypoxia and the activation of HIF-dependent gene networks is particularly robust in TNBC, targeting HIF directly might provide a new therapeutic option for patients with TNBC (13). There is no effective therapeutic agent readily available for TNBC at present. Therefore, more successful therapeutic strategies are required for breast cancer, such as TNBC, via the targeting of hypoxic conditions (14).

HIF is a crucial transcription factor that responds to hypoxic conditions. It transactivates a large number of genes involved in promoting angiogenesis, anaerobic metabolism and resistance to apoptosis. HIFs are heterodimers composed of one of three major oxygen-labile HIF- $\alpha$ subunits (HIF-1 $\alpha$, HIF- $2 \alpha$, or HIF-3 $\alpha$ ), and a constitutive HIF- $1 \beta$ subunit, which together form the HIF-1, HIF-2 and HIF-3 transcriptional complexes, respectively (15). Under aerobic conditions, HIF- $1 / 2 \alpha$ is hydroxylated by prolyl hydroxylases (PHDs) at two conserved proline residues in the oxygen-dependent degradation domain (ODD). The hydroxylation of HIF- $1 / 2 \alpha$ facilitates binding of the von Hippel-Lindau protein (pVHL) to HIF-1/2 $\alpha$ ODD, which causes poly-ubiquitination and proteasomal degradation of HIF-1/2 $\alpha$ (16). However, under hypoxic conditions, hydroxylation does not occur and HIF-1/2 $\alpha$ are stabilized and accumulate. HIFs then bind to a conserved DNA sequence known as the hypoxia response elements, and activate the transcription of a variety of hypoxia-responsive genes. The most potent proangiogenic growth factor, vascular endothelial growth factor (VEGF), is one of the HIF-1 $\alpha$-regulated genes and mediates hypoxia-driven angiogenesis. As a result of dysregulated and rapid cell proliferation, which is a characteristic of cancer cells and functionally abnormal blood vessels that form in solid tumors, the environment around cancer cells changes from normoxia $\left(21 \% \mathrm{O}_{2}\right)$ to hypoxia $\left(\sim 1 \% \mathrm{O}_{2}\right)(17,18)$. HIFs mediate the adaptation of cancer cells to an explicit hypoxic microenvironment. This mediation leads to VEGF expression, followed by the stimulation of angiogenesis, and thereby, increased $\mathrm{O}_{2}$ delivery. By repeating this process, hypoxic cancer cells acquire invasive and metastatic properties, as well as resistance to cancer therapy, which together constitute the lethal cancer phenotype. Given these factors, compounds that can inhibit HIF-1 may have the potential for use as anticancer agents.

Resveratrol (3,4,5-trihydroxy-trans-stilbene; Fig. 1A), a polyphenol derived from grapes and peanuts, has been shown to possess a wide range of health benefits, including cardioprotective, antioxidant, anti-inflammatory and anti-aging effects (19). Intense efforts over the past decades have indicated that resveratrol exhibits chemopreventive and therapeutic effects against a wide range of cancers (20). In spite of resveratrol's great anticancer potential, its utility as a therapeutic anticancer agent is limited by its relatively low bioavailability, photosensitivity and metabolic instability. Thus, numerous approaches are being undertaken to overcome these limitations and to obtain synthetic analogues superior to resveratrol.

HS-1793 [4-(6-hydroxy-2-naphthyl)-1,3-benzenediol; Fig. 1B], a novel synthetic resveratrol analogue, has been shown to exert stronger antitumor effects than those of resveratrol in a variety of cancer cell lines (21-25). Furthermore, it induced the modulation of tumor-derived $\mathrm{T}$ lymphocytes, especially in its suppressive role on the Treg cell population (26). It exhibits apoptogenic activity in a wide range of cancer cells, including breast $(21,22,27)$, prostate $(24)$, colon $(23,28)$, leukemia $(23)$ and renal carcinoma cells (25). The way in which this resveratrol analogue exerts its antiproliferative effects has not been fully elucidated. HS-1793 has been shown to induce G2/M cell cycle arrest (27), downregulate Bcl-2 and Bcl-xL expression (23-25,27), activate caspase pathways (27), induce endoplasmic reticulum stress-mediate apoptosis and inactivate Akt $(28,29)$. We have previously shown that this resveratrol analogue can inhibit hypoxia-induced HIF-1 $\alpha$ and VEGF expression in PC-3 prostate cancer cells via inhibition of phosphorylation of PI3K and Akt (30), and cause cell cycle arrest and apoptotic cell death in MCF-7 (hormone-dependent, wildtype p53) and MDA-MB-231 (TNBC, mutated p53) breast cancer cells (27). However, most of the studies investigating HS-1793's anticancer potential have been carried out in vitro. Only a limited number of animal studies have been conducted to reveal its anticancer activities.

In the present study, therefore, we used resveratrol and its synthetic analogue, HS-1793, to investigate and compare their effects on the expression of HIF-1 and VEGF in MCF-7 and MDA-MB-231 breast cancer cells in vitro and further anticancer effects in vivo with triple-negative MDA-MB-231 breast cancer xenografts in nude mice.

\section{Materials and methods}

Chemicals. Resveratrol and 3-(4,5-dimethylthiazol-2-yl)2,5-diphenyltetrazolium bromide (MTT), trypan blue stain and antibody against $\beta$-actin were purchased from Sigma-Aldrich (St. Louis, MO, USA). HS-1793 was synthesized and supplied by Professor Hongsuk Suh (Department of Chemistry, Pusan National University, Busan, Korea). A $100 \mathrm{mM}$ solution of resveratrol or HS-1793 was prepared in ethanol and stored in small aliquots at $-20^{\circ} \mathrm{C}$. The stock solution was diluted, when required, in cell culture medium. The maximum concentration of ethanol did not exceed $0.1 \%(\mathrm{v} / \mathrm{v})$ in the treatment range, at which it did not influence cell growth. Anti HIF-1 $\alpha$ was purchased from BD Transduction Laboratories (San Jose, CA, USA). Antibodies against VEGF and histone H1 were obtained from Santa Cruz Biotechnology (Dallas, TX, USA). Matrigel was purchased from BD Biosciences (San Jose, CA, USA).

Cell culture. The cancer cell lines used in the present study included human breast carcinoma (MCF-7 and MDA-MB-231) with normal human breast epithelial cell line (MCF-10A) serving as control. MCF-7 and MDA-MB-231 
were obtained from the American Type Culture Collection (ATCC; Manassas, VA, USA). MCF-10A cells were generously provided from Dr Robert J. Pauley (The Barbara Ann Karmanos Cancer Institute, Detroit, MI, USA). MCF-7 and MDA-MB-231 cells were cultured in Dulbecco's modified Eagle's medium (DMEM; HyClone Laboratories, Inc., Logan, UT, USA) containing $10 \%$ heat-inactivated fetal bovine serum (FBS; HyClone Laboratories) and $1 \%$ antibiotic-antimycotic solution (HyClone Laboratories) at $37^{\circ} \mathrm{C}$ in a humidified atmosphere with $5 \% \mathrm{CO}_{2}$, not exceeding passage number 20 . MCF-10A cells were maintained in DMEM/F-12 (1:1) with 5\% horse serum (Thermo Fisher Scientific, Waltham, MA, USA) in $37^{\circ} \mathrm{C}$ incubator supplied with $5 \% \mathrm{CO}_{2}$.

Hypoxia experiments. Experiments to investigate the effects of hypoxia were carried out in a hypoxia chamber in an anaerobic system (Thermo Fisher Scientific, Marietta, OH, USA) as previously described (30). Hypoxic conditions were designated as $1 \% \mathrm{O}_{2}$ and $5 \% \mathrm{CO}_{2}$ with the temperature maintained at $37^{\circ} \mathrm{C}$. Normoxia was defined as the conditions in a standard $\mathrm{CO}_{2}$ incubator $\left(21 \% \mathrm{O}_{2}\right.$ and $\left.5 \% \mathrm{CO}_{2}\right)$. For hypoxia experiments, MCF-7 and MDA-MB-231 cells were grown to 50\% confluency in a standard $\mathrm{CO}_{2}$ incubator at $37^{\circ} \mathrm{C}$. Twenty-four hours prior to experiments, aliquots of cell culture media were placed in normoxic and hypoxic chambers to allow equilibration to the corresponding conditions. Immediately before each experiment, cell culture media were withdrawn from MCF-7 and MDA-MB-231 cells and replaced with equilibrated media.

MTT assay and growth inhibition. Cell viability was determined by a colorimetric MTT assay as previously described (31). Briefly, cells were seeded onto 6-well plates at a density of $2 \times 10^{5}$ cells/well and allowed to adhere and grow overnight. Cells were then treated with increasing concentrations of resveratrol, HS-1793, or ethanol vehicle for $24 \mathrm{~h}$ in normoxic condition or for $4 \mathrm{~h}$ in hypoxic condition. Fresh medium with MTT was added to the wells, and the plate was incubated at $37^{\circ} \mathrm{C}$ for $2 \mathrm{~h}$. The medium was discarded, the formazan crystals were dissolved in dimethyl sulfoxide, and the absorbance at $540 \mathrm{~nm}$ was measured using an ELISA plate reader (Thermo Fisher Scientific, Vantaa, Finland). The ethanol vehicle-treated cells served as the indicator of $100 \%$ cell viability. Percentage of cell viability was calculated using the following calculation formula: Cell viability $(\%)=(\mathrm{OD}$ sample/OD control) x $100 \%$. IC S0 $_{50}$ value (concentration of resveratrol or HS-1793 that reduce $50 \%$ cell viability compared to ethanol vehicle-treated control cell) was determined from the graph of viability (\%) vs. resveratrol or HS-1793 concentration ranging between 100 and $12.5 \mu \mathrm{M}$ by 2 -fold serial dilution. All cell lines were assayed for three biological replicates each with triplicates.

Western blot analysis. Cells were homogenized in protein lysate buffer, and the debris was removed by centrifugation at $12,000 \mathrm{rpm}$ for $10 \mathrm{~min}$ at $4^{\circ} \mathrm{C}$. The nuclear and cytosolic fractions from tumor tissue were prepared as previously described (32). The protein concentrations in all samples were determined by protein assay reagents (Bio-Rad Laboratories, Hercules, CA, USA). Equal quantities of proteins were subjected to sodium dodecyl sulfate-polyacrylamide gel electrophoresis. They were then transferred onto polyvinylidene fluoride membranes, and blocked with $5 \%$ non-fat dried milk for $1 \mathrm{~h}$ at $37^{\circ} \mathrm{C}$. The membranes were probed with specific primary antibodies overnight at $4^{\circ} \mathrm{C}$, and then incubated with the corresponding secondary antibodies for $1 \mathrm{~h}$ at $37^{\circ} \mathrm{C}$. The specific protein bands were visualized with an ECL detection system (GE Healthcare, Piscataway, NJ, USA).

VEGF ELISA. To analyze VEGF expression quantitatively, MCF-7 and MDA-MB-231 cells were pretreated with resveratrol, HS-179, or vehicle for $30 \mathrm{~min}$. The treatment was then removed and replaced with fresh media, which were preconditioned in normoxic or hypoxic conditions. Cells were incubated in the presence or absence of resveratrol or HS-1793 at corresponding conditions for $24 \mathrm{~h}$. The supernatants in the wells were collected, cleared by centrifugation and stored at $-20^{\circ} \mathrm{C}$. ELISA was performed using the human VEGF Quantikine kit (R\&D Systems, Minneapolis, MN, USA) according to the manufacturer's suggested protocol.

$R N A$ extraction and RT-PCR. Total RNA isolated from breast cancer cells using a RNeasy Mini kit reagent (Qiagen, La Jolla, CA, USA), was reverse transcribed using a Bioneer RT/PCR PreMix in the presence of oligo dT (Bioneer Corp., Daejeon, Korea). The resulting complementary DNA was amplified with the following sets of oligonucleotide primers: VEGF (sense, 5'-AGGAGGGCAGAATCATCACG-3' and antisense, 5'-CAAGGCCCACAGGGATTTTCT-3'), and glyceraldehyde3-phosphate dehydrogenase (GADPH; sense, 5'-CGGAGT CAACGGATTTGGTCGTAT-3' and antisense, 5'-AGCCTTC TCCATGGTGGTGAAGAC-3'). GAPDH served as an internal control. PCR products were analyzed by electrophoresis on a $1.5 \%$ agarose gel (Bio Basic, Inc., Markham, ON, Canada) in the presence of ethidium bromide, and were visualized with a

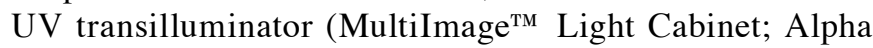
Innotech Corp., San Leandro, CA, USA).

Animal studies. The animal protocol used in the present study was reviewed and approved by the Pusan National University-Institutional Animal Care and Use Committee (PNU-IACUC, Busan, Korea) in terms of ethical procedures and scientific care (approval number, PNU-2015-0318). Five-week-old female BALB/c nude mice (Japan SLC, Inc., Hamamatsu, Japan) were used for in vivo experiments. The animals were maintained in constant, specific pathogenfree laboratory conditions for a $12 \mathrm{~h}$ light/dark cycle. They were given water and fed standard mouse chow ad libitum. For injections, MDA-MB-231 cells were trypsinized and counted using trypan blue to identify viable cells. Animals were injected with $1 \times 10^{6}$ MDA-MB-231 cells [in $100 \mu 1$ of phosphate buffered saline (PBS) and Matrigel, 1:1] in the right flank and allowed to form xenografts. When the average tumor volume reached $40 \mathrm{~mm}^{3}$, mice were randomly assigned to one vehicle and three treatment groups (6 mice per group): i) vehicle; ii) HS-1793 (5 mg/kg); iii) HS-1793 (10 mg/kg); and iv) resveratrol $(20 \mathrm{mg} / \mathrm{kg})$. HS-1793 and resveratrol were dissolved in PBS containing $0.1 \% \mathrm{v} / \mathrm{v}$ dimethyl sulfoxide (DMSO) and administered intraperitoneally twice a week. Tumor diameters were determined with a caliper, and the tumor volume was calculated using a standard formula: tumor 
Table I. The values of $\mathrm{IC}_{50}$ of resveratrol or HS-1793 in MCF-7, MDA-MB-231 and MCF-10A. ${ }^{a}$

\begin{tabular}{lcc}
\hline Cells & Resveratrol $(\mu \mathrm{M})$ & HS-1793 $(\mu \mathrm{M})$ \\
\hline MCF-7 & $88.2 \pm 4.7$ & $26.3 \pm 3.2$ \\
MDA-MB-231 & $90.6 \pm 2.9$ & $48.2 \pm 4.2$ \\
MCF-10A & $>100$ & $>100$ \\
\hline
\end{tabular}

${ }^{a}$ Exponentially growing MCF-7, MDA-MB-231 and MCF-10A cells at $60-70 \%$ confluence were treated with resveratrol or HS-1793 (12.5, $25,50$ and $100 \mu \mathrm{M})$ for $24 \mathrm{~h}$ and MTT assay was performed to calculate the $\mathrm{IC}_{50}$ values.

volume $\left(\mathrm{mm}^{3}\right)=L 1 \times(L 2)^{2} \times 0.5236$, where $L 1$ is the long diameter and $L 2$ is the short diameter. Toxicity was assessed by survival, activity and changes in body weight. At the completion of 4 weeks of treatment, the mice were euthanized and tumor samples were dissected out, weighed, fixed in formalin, and processed to determine the expression of target proteins as described in the sections on immunohistochemical analysis and western blotting.

Immunohistochemical analysis of tumors. Tumor tissues were fixed in $10 \% \mathrm{v} / \mathrm{v}$ neutral buffered formalin, embedded in paraffin, sectioned to $5 \mu \mathrm{m}$ and mounted on slides. The sections were blocked with normal goat serum and incubated with the following antibodies; anti-CD31 (Abcam, Cambridge, MA, USA) and anti-Ki-67 (Abcam). Stained slides were visualized with an Axiovert 100 microscope (Zeiss Carl, Göettingen, Germany) and the images were captured at a x 200 magnification.

Statistical analyses. Results were expressed as the mean \pm standard deviation (SD) of three separate experiments and analyzed using the Student's t-test. Means were considered significantly different at $\mathrm{P}<0.05$ or $\mathrm{P}<0.01$.

\section{Results}

HS-1793 suppresses proliferation of MCF-7 and MDA-MB-231 cells. We examined the effects of resveratrol or HS-1793 on the viability of MCF-7, MDA-MB-231 and MCF-10A cells cultured in normoxic condition for $24 \mathrm{~h}$. Table I summarized the $\mathrm{IC}_{50}$ value of resveratrol and HS-1793 on all the tested cells. The $\mathrm{IC}_{50}$ values in MCF-7, MDA-MB-231 and MCF-10A cells treated with resveratrol were $88.2 \pm 4.7,90.6 \pm 2.9$ and $>100 \mu \mathrm{M}$, respectively. The $\mathrm{IC}_{50}$ values in MCF-7 and MDA-MB-231 cells treated with HS-1793 were $26.3 \pm 3.2,48.2 \pm 4.2$ and $>100 \mu \mathrm{M}$, respectively. Therefore, HS-1793 treatment exhibited 3.3- and 1.9-fold more anti-proliferative effects against MCF-7 and MDA-MB-231 cells than resveratrol. However, no significant differences were observed between resveratrol and HS-1793 against the non-malignant normal MCF-10A cells.

HS-1793 inhibits hypoxia-induced HIF-1a protein in MCF-7 and MDA-MB-231 cells. To examine the effects of resveratrol and HS-1793 on HIF-1 $\alpha$ expression, we first exposed breast
A
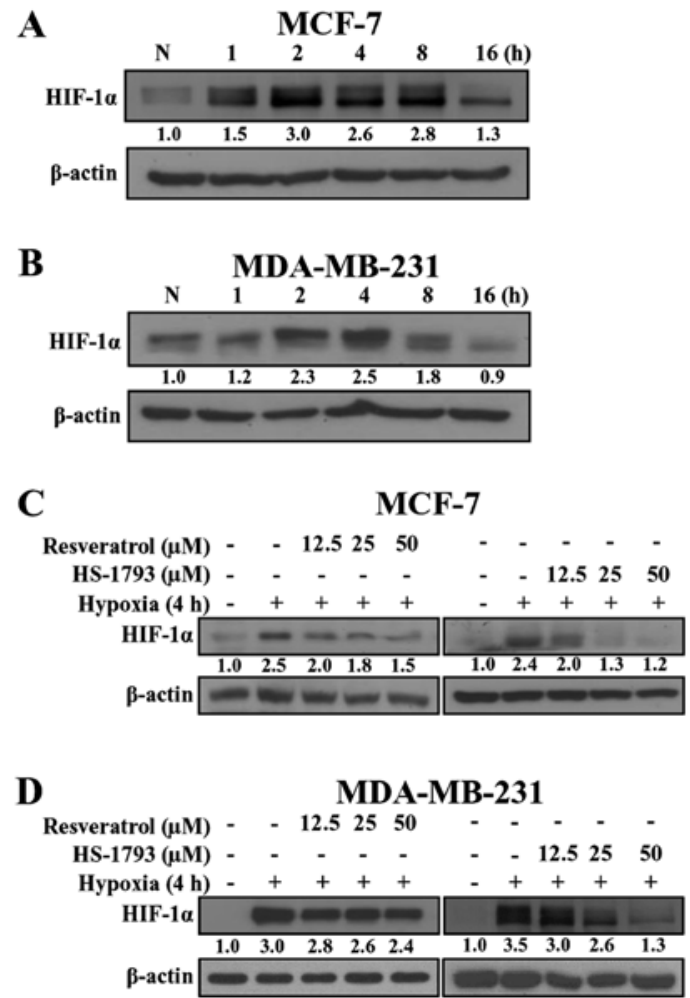

Figure 2. Inhibition of hypoxia-induced hypoxia-inducible factor (HIF)-1 $\alpha$ protein expression by HS-1793 in human breast cancer cells. (A) MCF-7 and (B) MDA-MB-231 cells were incubated in a hypoxic chamber for the indicated time. Western blot analysis was used to detect HIF-1 $\alpha$ protein expression. (C) MCF-7 and (D) MDA-MB-231 cells were exposed to $1 \% \mathrm{O}_{2}$ in combination with various concentrations of resveratrol or HS-1793 for $4 \mathrm{~h}$. Cells were lysed, and the expression of HIF-1 $\alpha$ protein was detected by western blot analysis. Protein levels were normalized to that of $\beta$-actin. Densitometry values are expressed as the fold-change compared with samples acquired in normoxia $(\mathrm{N})$ condition. Representative results from three independent experiments are shown. $\beta$-actin was used as a loading control. $\mathrm{N}$, normoxia.

cancer cells to hypoxic conditions and measured the HIF-1 $\alpha$ protein level to determine the optimum conditions. As shown in Fig. 2, hypoxia induced the expression of HIF-1 $\alpha$ in MCF-7 and MDA-MB-231 cells in a time-dependent manner.In MCF-7 cells (Fig. 2A), the induction of HIF-1 $\alpha$ protein was observed within $1 \mathrm{~h}$ after switching to hypoxic conditions, and activation continued for $16 \mathrm{~h}$. Unlike MCF-7 cells, in MDA-MB-231 cells the expression of HIF-1 $\alpha$ started at $2 \mathrm{~h}$, reached a maximum at $4 \mathrm{~h}$, and decreased at $8 \mathrm{~h}$ (Fig. 2B). Since both breast cancer cell lines exhibited the highest expression of HIF-1 $\alpha$ at 2-4 h, we used the $4 \mathrm{~h}$ time-point in subsequent experiments.

We next determined whether resveratrol and HS-1793 could modulate the expression of HIF-1 $\alpha$ protein under hypoxic conditions. As shown in Fig. $2 \mathrm{C}$ and D, both resveratrol and HS-1793 downregulated HIF-1 $\alpha$ expression in a concentration-dependent manner. Notably, HS-1793 more effectively decreased the level of HIF-1 $\alpha$ when compared to resveratrol in both cell lines. In addition, a significant decrease in the HIF- $1 \alpha$ level was demonstrated in response to resveratrol and HS-1793 in MDA-MB-231 cells (Fig. 2D).

Decrease of HIF-1 a protein levels by HS-1793 is unrelated to cell death. To investigate whether resveratrol and 

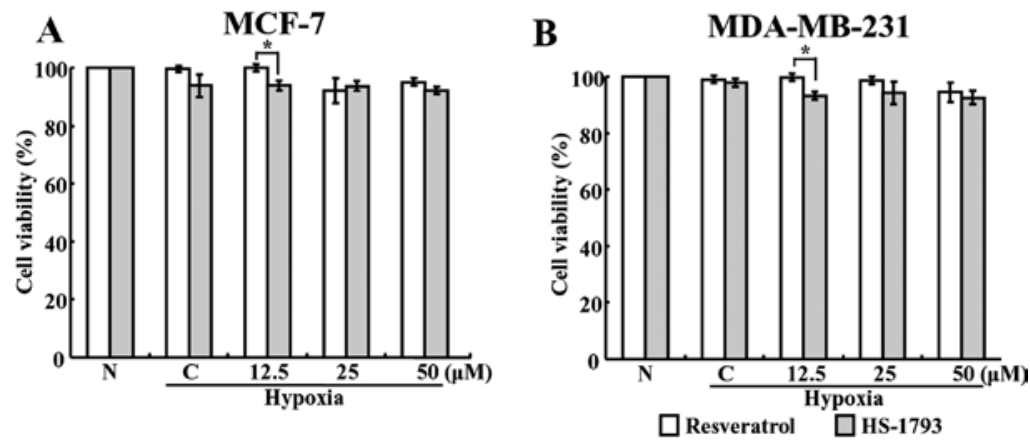

Figure 3. Effects of resveratrol and HS-1793 on cell viability. Cells were treated with resveratrol or HS-1793 for $4 \mathrm{~h}$ under (A) normoxic or (B) hypoxic conditions. Cell viability was determined by MTT assay. Results are expressed as percentages of the vehicle-treated control \pm SD of three independent experiments. The significance was determined by Student's t-test ( $\mathrm{P}<0.05$ comparison between resveratrol- and HS-1793-treated cells). N, normoxia; $\mathrm{C}$, vehicle-treated control.

A

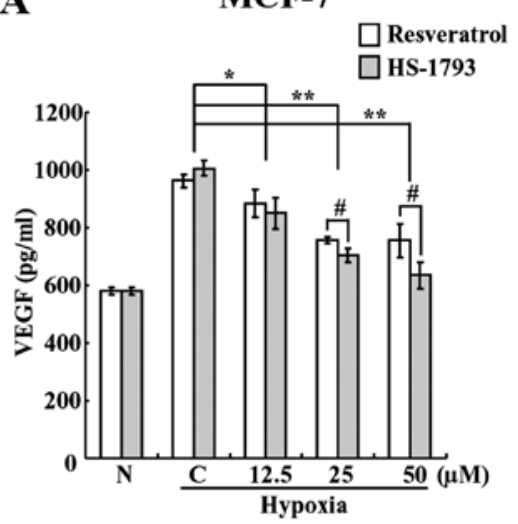

B

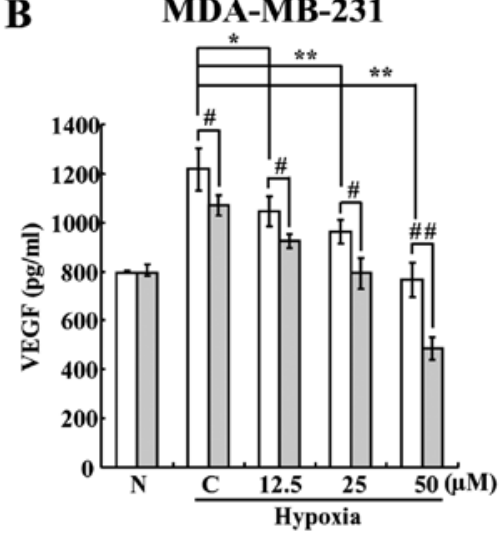

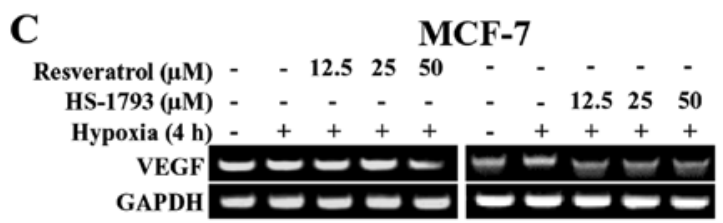

D

MDA-MB-231

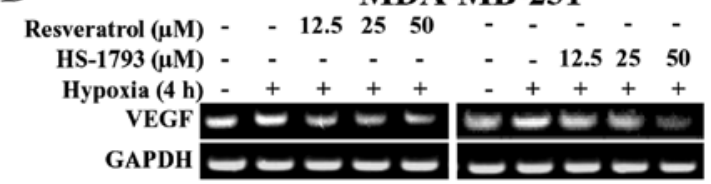

Figure 4. Suppression of vascular endothelial growth factor (VEGF) production by HS-1793 in human breast cancer cells maintained in hypoxic conditions. (A) MCF-7 and (B) MDA-MB-231 cells were treated with various concentrations of resveratrol or HS-1793 for $24 \mathrm{~h}$ in hypoxic conditions, and the VEGF level in the culture media was quantitatively determined using ELISA. The assay was performed in triplicate, and results are reported as the mean \pm SD. "P $<0.05$, ${ }^{* *} \mathrm{P}<0.01$ vs. vehicle-treated control; ${ }^{\#} \mathrm{P}<0.05$ and ${ }^{\# \#} \mathrm{P}<0.01$ compared between resveratrol- and HS-1793-treated cells. N, normoxia; $\mathrm{C}$, vehicle-treated control. (C) MCF-7 and (D) MDA-MB-231 cells were exposed to $1 \% \mathrm{O}_{2}$ and treated with various concentrations of resveratrol or HS-1793 for $4 \mathrm{~h}$. VEGF mRNA levels were determined by reverse transcription-polymerase chain reaction analysis. Representative results from three independent experiments are shown. GAPDH was used as a loading control.

HS-1793-induced cell death was responsible for the suppression of HIF-1 $\alpha$ accumulation, the cell viabilities in normoxic and hypoxic conditions were determined using the MTT assay. When both cells were treated with various concentrations of resveratrol and HS-1793 for $4 \mathrm{~h}$ in hypoxic conditions, no significant reductions in the viability was observed at any concentration (Fig. 3). These results suggest that the decrease in HIF-1 $\alpha$ under hypoxic conditions may not be due to cell death.
HS-1793 downregulates hypoxia-induced VEGF expression in breast cancer cells. VEGF is one of the downstream target genes of HIF-1 $\alpha$; therefore, its level increases under hypoxic conditions, and it plays a crucial role in tumor angiogenesis (33). Therefore, we investigated whether HS-1793 inhibits VEGF under hypoxic conditions. In order to do this, we first performed ELISA to determine whether HS-1793 and resveratrol affect VEGF levels in breast cancer cells. As expected, hypoxia caused a rise in the VEGF level when compared 

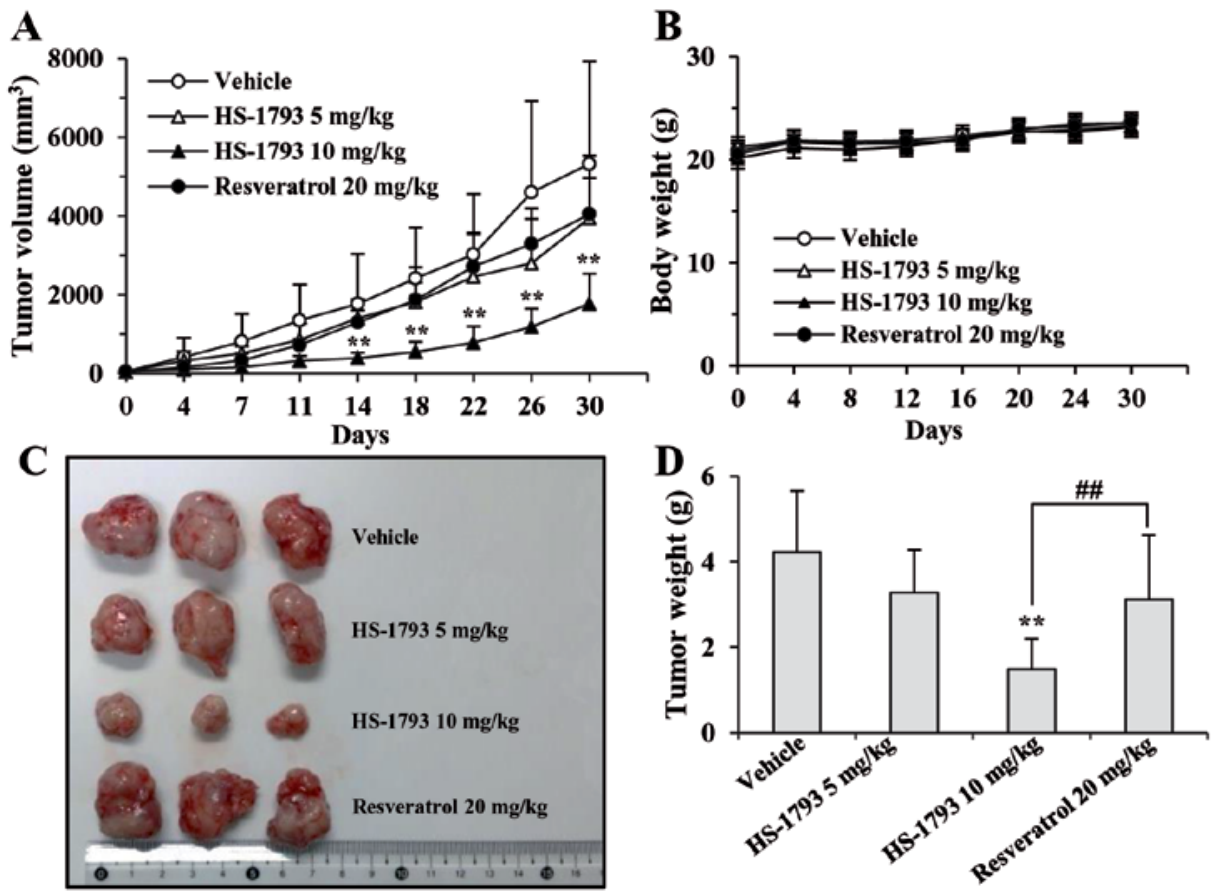

Figure 5. Inhibition of breast cancer xenograft growth by HS-1793 in vivo. Mice were subcutaneously injected with MDA-MB-231 cells (1x10 ${ }^{6}$ cells $/$ mouse) mixed with Matrigel (1:1). Once the tumors reached $\sim 40 \mathrm{~mm}^{3}$, the mice were treated with intraperitoneal injections of either the vehicle control $(0.1 \% \mathrm{v} / \mathrm{v}$ DMSO in PBS), HS-1793 (5 or $10 \mathrm{mg} / \mathrm{kg}$ ) or resveratrol $(20 \mathrm{mg} / \mathrm{kg}$ ) twice a week. After 4 weeks of treatment, the mice were sacrificed. Tumor measurements were taken using a caliper and were then used to calculate the tumor volume (A). Changes in body weight (B). Tumors were removed and photographed (C). Tumors were weighed at the end of study (D). Results are reported as the mean $(\mathrm{n}=6) \pm \mathrm{SD}$. ${ }^{* *} \mathrm{P}<0.01$ compared with the vehicle-treated control. ${ }^{\# \#} \mathrm{P}<0.01$ compared between resveratrol- and HS-1793-treated groups.

with normoxia (Fig. 4A and B). However, the expression levels of VEGF were decreased in a concentration-dependent manner following treatment with resveratrol and HS-1793 treatments (Fig. 4A and B). Moreover, HS-1793 reduced the VEGF level to a greater extent than resveratrol in both cell types. These results showed that HS-1793 is more effective than resveratrol at inhibiting the production of VEGF in both cancer cell lines. In addition, HS-1793 treatment at $50 \mu \mathrm{M}$ concentration more efficiently reduced the expression levels of VEGF in MDA-MB-231 cells than in MCF-7 cells.

HS-1793 suppresses hypoxia-induced mRNA expression of $V E G F$ at the transcriptional level.In order to determine whether HS-1793 induces a reduction of VEGF through suppressing the expression of VEGF, we determined its mRNA levels under hypoxic conditions using reverse transcription-polymerase chain reaction (RT-PCR). Breast cancer cells were pretreated with various concentrations of resveratrol or HS-1793 and incubated for $4 \mathrm{~h}$ under hypoxic conditions, and the mRNA levels were measured. As shown in Fig. 4C and D, HS-1793 downregulated the expression of $V E G F$ mRNA, with the more marked results observed in MDA-MB-231 cells. In MCF-7 cells, the effect was observed at a concentration of $12.5 \mu \mathrm{M}$ HS-1793, a concentration at which resveratrol had no significant effects on the mRNA expression of VEGF (Fig. 4C). In addition, we saw the suppression of $V E G F$ mRNA by resveratrol only at the highest concentration $(50 \mu \mathrm{M})$ used in this study. We also found that resveratrol slightly inhibited the mRNA expression of $V E G F$ in MDA-MB-231 cells, whereas HS-1793 highly effective at inhibiting $V E G F$ mRNA expression in a concentration-dependent manner (Fig. 4D). Neither resveratrol nor HS-1793 had any effect on the mRNA expression of $H I F-1 \alpha$ under these experimental conditions (data not shown). Therefore, these results indicate that the inhibition of VEGF expression by HS-1793 occurs at the transcriptional level. In summary of in vitro experiments, HS-1793 treatments showed more efficient downregulation of HIF-1 $\alpha$ and VEGF expression levels in MDA-MB-231 cells than in MCF-7 cells, we decided to use TNBC MDA-MD-231 cells for further in vivo xenograft experiment.

HS-1793 effectively inhibits the growth of human breast xenografts. To explore the therapeutic effects of HS-1793 as a potentially clinically useful agent, we compared the in vivo efficacy of resveratrol and its synthetic analogue, HS-1793, in TNBC MDA-MB-231 tumor-bearing mice. As shown in Fig. 5A, tumor growth was rapid in the vehicle-treated control group, whereas HS-1793 significantly inhibited MDA-MB-231 xenograft tumor growth in a dose-dependent manner. Notably, the tumor-inhibitory effects of low-dose HS-1793 $(5 \mathrm{mg} / \mathrm{kg}$ twice a week) and a 4 -fold higher dose $(20 \mathrm{mg} / \mathrm{kg}$ twice a week) of the parent agent, resveratrol, were similar, although no statistical significance was observed (Fig. 5A, C and D). HS-1793 (5 mg/kg twice a week) showed 2-fold higher maximum growth inhibition when compared to resveratrol (Fig. 5A and D), indicating that the in vivo efficacy of HS-1793 was superior to that of resveratrol. In addition, there were no adverse side-effects, such as weight loss, ulcerations, or general decreases in well-being in the drug-treated mice in comparison to the vehicle-treated control group during the experimental period. This indicates the non-toxicity of both resveratrol and HS-1793 (Fig. 5B). 

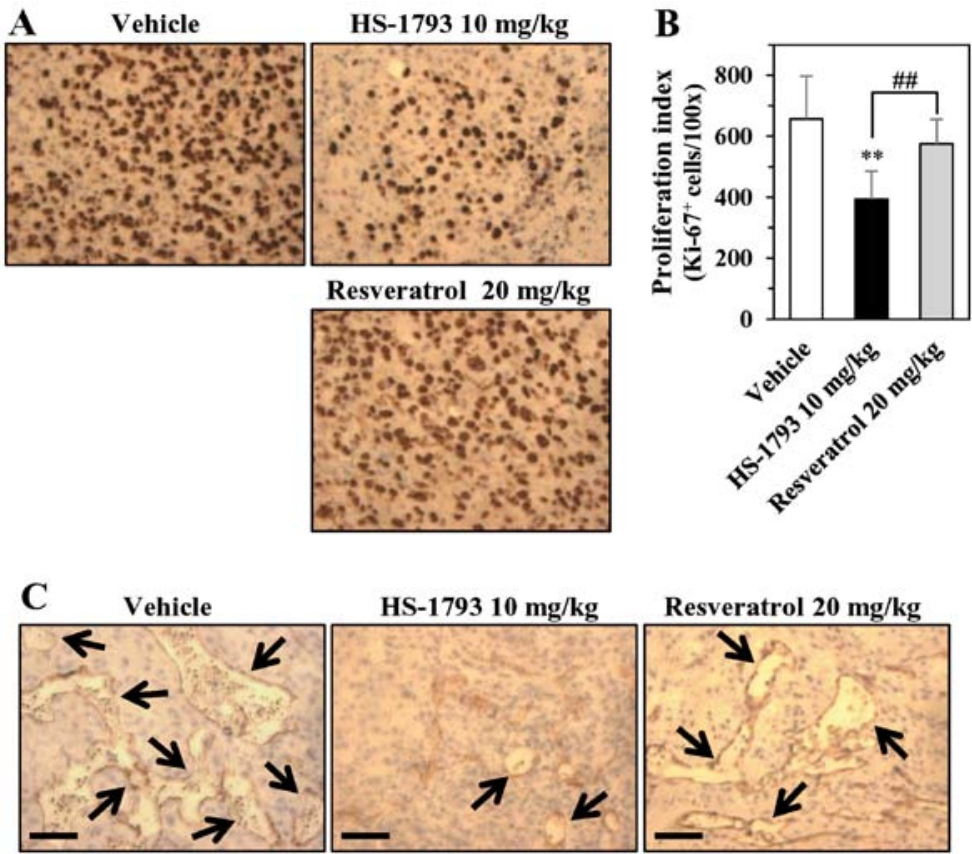

Figure 6. Suppression of tumor proliferation and inhibition of vessel formation by HS-1793 in MDA-MB-231 xenograft. Immunohistochemical (IHC) staining of tumor tissues for the proliferation marker Ki-67 (A). Representative Ki- $67^{+}$IHC images are shown (left three panels). A total of five x100 fields were examined from three tumors in each of the treatment groups (right panel). Results of proliferation index are displayed as the mean $\pm \mathrm{SD}(\mathrm{B}){ }^{* *} \mathrm{P}<0.01 \mathrm{compared}$ with the vehicle-treated control. ${ }^{\# \#} \mathrm{P}<0.01$ compared between resveratrol- and HS-1793-treated groups. IHC staining of tumor tissues for vessel formation marker CD31 from vehicle-treated mice and mice treated with HS-1793 or resveratrol (C). Sections of tumors showed capillaries (marked as arrows). Bar, $100 \mu \mathrm{m}$.
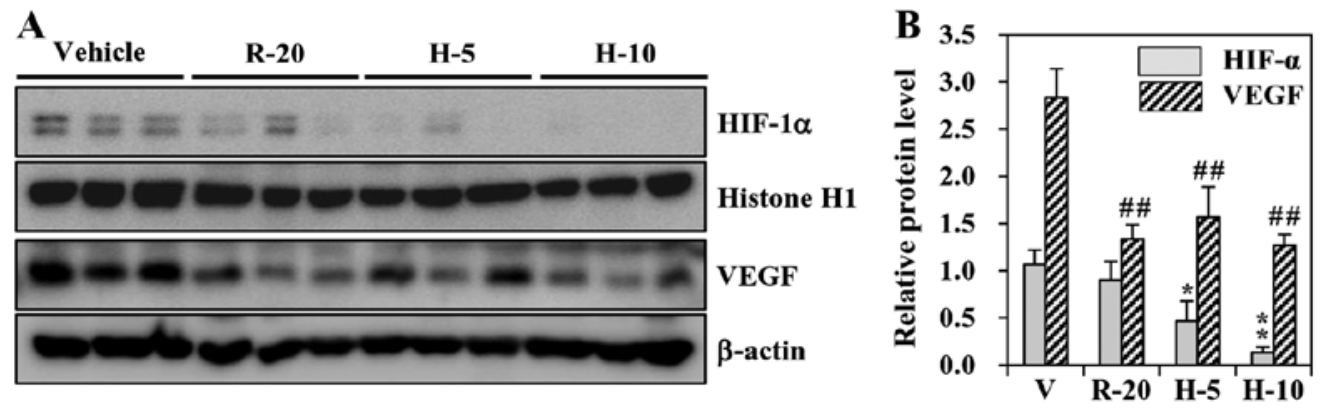

Figure 7. Suppression of HIF-1 and VEGF expression by HS-1793 in MDA-MB-231 xenografts. (A) Protein extracts prepared from tumors from at least three different mice were analyzed by western blot analysis by using antibodies against HIF-1 $\alpha$ and VEGF. Histone H1 was used as a loading control for nuclear extracts. $\beta$-actin was used as a loading control for cytoplasmic extracts. (B) The bar graphs represent fold-changes in HIF-1 $\alpha$ and VEGF protein expression (mean \pm SD) in MDA-MB-231 xenograft tumors from at least three different mice. ${ }^{*} \mathrm{P}<0.05,{ }^{* * *} \mathrm{P}<0.01,{ }^{\# \prime} \mathrm{P}<0.01$ vs. vehicle-treated control. H-5, HS-1793 $5 \mathrm{mg} / \mathrm{kg} ; \mathrm{H}-10$, HS-1793 $10 \mathrm{mg} / \mathrm{kg}$; R-20, resveratrol $20 \mathrm{mg} / \mathrm{kg} ; \mathrm{V}$, vehicle-treated control.

To shed light on the mechanism by which HS-1793 inhibited tumor growth in nude mice, we examined the presence of proliferation markers in tumor tissues from vehicle- and HS-1793-treated groups. Ki-67 (a proliferation marker) expression was significantly lower in the HS-1793-treated group than in the vehicle-control group (Fig. 6A). As shown in Fig. 6A (right panel), HS-1793 was more effective than resveratrol (Fig. 6A, bottom panel) in downregulating Ki-67 levels. The results of proliferation index were summarized in Fig. 6B. We also examined the effects of HS-1793 and resveratrol on tumor-associated angiogenesis, as this process is critical for tumor survival and proliferation (34). Change in angiogenesis was validated using immunohistochemistry of CD31 expression in the sections of tumors. Results showed that as compared to vehicle-treated and resveratrol-treated tumors, the expression of $\mathrm{CD} 31$ was significantly lower in tumors treated with HS-1793 (Fig. 6C). This result suggests relatively hampered angiogenesis in HS-1793-treated tumors, which may be contributing in the slower tumor growth in this group of mice.

HS-1793 inhibits HIF-1 and VEGF expression in xenograft tumors from mice. Up to this point, our results indicated that HS-1793 can inhibit the proliferation and vessel formation which linked to tumor development. We next sought to determine the effects of resveratrol and HS-1793 treatment on the expression of HIF-1 $\alpha$ and its-regulated gene product VEGF in tumor tissue. As shown in Fig. 7, the tumor tissues from vehicle-treated mice groups expressed HIF-1 $\alpha$ and VEGF. Treatments of resveratrol $(20 \mathrm{mg} / \mathrm{kg}$ ) and HS-1793 (5 and 
$10 \mathrm{mg} / \mathrm{kg}$ ) successfully suppressed the expression of HIF-1 $\alpha$ and VEGF in tumor tissues. The results also indicated that the tumor tissues expressed VEGF (Fig. 7A). However, HS-1793 is more effective than resveratrol in suppressing the expression of HIF-1 $\alpha$ and VEGF in vivo (Fig. 7B).

\section{Discussion}

Numerous studies have suggested that the transcription factor HIF-1 $\alpha$ is a crucial mediator of the hypoxic response, which plays a role in triggering tumor metastasis and developing chemoresistance in cancer cells. HIF-1 $\alpha$ overexpression is closely associated with the unfavorable prognosis and increased mortality in cancer patients $(35,36)$. Thus, new agents that target this transcription factor have gained attention. The goal of the present study was to determine whether the novel resveratrol analogue HS-1793 could inhibit HIF-1 $\alpha$, which is closely linked with cancer cell proliferation, invasion and angiogenesis.

Our results showed that HS-1793 reduced the protein level of HIF-1 $\alpha$ without affecting its mRNA level, therefore, suggesting that its action occurs at the post-transcriptional level. We found that HS-1793 activates the ubiquitin-proteasome pathway, which is responsible for HIF-1 $\alpha$ protein degradation (data not shown). This is evidenced by the fact that the $26 \mathrm{~S}$ proteasome-specific protease inhibitor, MG132, is able to delay the degradation of HIF- $1 \alpha$ in the presence of HS-1793. This is further supported by our previous report that showed HS-1793 promoted the degradation of HIF- $1 \alpha$ via the ubiquitinproteasome pathway in prostate cancer cells (30). However, a recent study has documented that the autophagy-lysosome pathway involves degradation of the HIF-1 $\alpha$ protein (37), thus, HS-1793 could induce autophagy in cancer cells (unpublished data). The precise mechanism by which HS-1793 regulates HIF- $1 \alpha$ needs to be elucidated in future studies.

This study demonstrated that HS-1793 decreased hypoxiainduced mRNA expression and secretion of VEGF in breast cancer cells, which is in agreement with our previous finding in prostate cancer cells (30). VEGF is known to be a major signaling molecule involved in tumor angiogenesis, and is regulated by HIF-1 $\alpha$ (38). Therefore, the observed inhibition of HIF-1 $\alpha$ may have accounted for the downregulation of VEGF by HS-1793. We also found that HS-1793 decreased angiogenesis in vivo, as indicated by the inhibition of CD31, a marker for microvessel density and the suppression of VEGF. The downregulation of the levels of these proteins suggests that this resveratrol analogue possesses anti-angiogenic potential.

Accumulating evidence indicates that resveratrol requires relatively high doses and frequent injections to exhibit its tumor growth inhibitory effect. This is due to its poor bioavailability, as a result of its low intestinal uptake and short initial halflife (39-42). Furthermore, there has been controversy about the antitumor effects of resveratrol on mammary tumors, with early reports showing that resveratrol $(25 \mathrm{mg} / \mathrm{kg}$ bodyweight, intraperitoneal injection) inhibited the growth of MDA-MB-231 tumors (43). However, Castillo-Pichardo et al (44) recently reported that a range of concentrations $(0.5,5$ and $50 \mathrm{mg} / \mathrm{kg}$ body weight) of resveratrol promotes mammary tumor growth in mice. We found that HS-1793 at $5 \mathrm{mg} / \mathrm{kg}$ showed a modest inhibitory effect on the growth of breast cancer in a xenograft implanted nude mouse model, with a further enhancement of the antitumor effects at $10 \mathrm{mg} / \mathrm{kg}$. A significant $70 \%$ reduction in tumor growth was observed at $10 \mathrm{mg} / \mathrm{kg}$ HS-1793 when compared with growth in the vehicle control. This growthinhibitory effect of HS-1793 occurred in a dose-dependent manner. In addition, all animals tolerated HS-1793 very well, as indicated by the lack of significant body weight differences between agent-treated and vehicle-treated mice from the start of the study. HS-1793, as low as $5 \mathrm{mg} / \mathrm{kg}$ of body weight, inhibited tumor growth (volume) by $26 \%$ when compared with the vehicle-control, which was similar to that of resveratrol at a dose of $20 \mathrm{mg} / \mathrm{kg}$. These results were further corroborated by earlier studies showing that HS-1793 exerted a considerable effect on the in vivo growth of an FM3A breast tumor in $\mathrm{CH} 3 / \mathrm{He}$ mice (26). Furthermore, at a comparable dose of resveratrol, it neither inhibited the growth, nor suppressed metastasis in 4T1 breast cancer-bearing mice (45).

Although we showed, for the first time, that HS-1793 had therapeutic effects against human breast cancer xenografts; there are some reports about the anticancer effects of this resveratrol analogue in vivo. Previously, HS-1793 has been shown to have therapeutic effects on established tumors in FM3A tumor-bearing mice via the suppression of Treg cells (26). The same study also showed the chemopreventive effect of HS-1793 (26). Jeong et al (46) showed that HS-1793-administration increased the number of interferon (IFN)- $\gamma$-secreting cells in splenocytes, which lead to the switch-off of M-2 polarized tumor-associated macrophages with immunosuppressive and tumor progressive properties, which likely contributes the antitumor effect of HS-1793.

In conclusion, we provide novel evidence that HS-1793 exhibits its anticancer activity, at least in part, by modulating HIF- $1 \alpha$ and its regulating gene, VEGF. In our xenograft mouse study with TNBC MDA-MB-231 cells, which bear an aggressive phenotype, HS-1793 not only inhibited tumor growth, but also suppressed microvessel formation, which strongly correlated with the inhibition of cell proliferation, and the decrease in angiogenesis. These findings provide a rationale for further investigation into this novel resveratrol analogue for chemoprevention and/or treatment in human breast cancer.

\section{Acknowledgements}

The present study was supported by the Basic Science Research Program through the National Research Foundation of Korea (NRF), and was funded by the Ministry of Education, Science, and Technology (2012R1A1A2006753, 2014R1A1A2055336). This study was also supported by the National Research Foundation of Korea (NRF) grant funded by the Korea government (MSIP) (No. 2009-0083538). We thank Aging Tissue Bank for providing research information. This study was also financially supported by the 2016 Post-Doc. Development Program of Pusan National University.

\section{References}

1. Torre LA, Islami F, Siegel RL, Ward EM and Jemal A: Global cancer in women: Burden and trends. Cancer Epidemiol Biomarkers Prev 26: 444-457, 2017.

2. Brenton JD, Carey LA, Ahmed AA and Caldas C: Molecular classification and molecular forecasting of breast cancer: Ready for clinical application? J Clin Oncol 23: 7350-7360, 2005. 
3. Anders CK and Carey LA: Biology, metastatic patterns, and treatment of patients with triple-negative breast cancer. Clin Breast Cancer 9 (Suppl 2): S73-S81, 2009.

4. Yadav BS, Sharma SC, Chanana P and Jhamb S: Systemic treatment strategies for triple-negative breast cancer. World J Clin Oncol 5: 125-133, 2014.

5. Tao M, Ma D, Li Y, Zhou C, Li Y, Zhang Y, Duan W, Xu X, Wang R, Wu L, et al: Clinical significance of circulating tumor cells in breast cancer patients. Breast Cancer Res Treat 129: 247-254, 2011

6. Voss MJ, Möller MF, Powe DG, Niggemann B, Zänker KS and Entschladen F: Luminal and basal-like breast cancer cells show increased migration induced by hypoxia, mediated by an autocrine mechanism. BMC Cancer 11: 158, 2011.

7. Pouysségur J, Dayan F and Mazure NM: Hypoxia signalling in cancer and approaches to enforce tumour regression. Nature 441 437-443, 2006

8. Wilson WR and Hay MP: Targeting hypoxia in cancer therapy. Nat Rev Cancer 11: 393-410, 2011.

9. Semenza GL: Oxygen sensing, hypoxia-inducible factors, and disease pathophysiology. Annu Rev Pathol 9: 47-71, 2014.

10. Semenza GL: The hypoxic tumor microenvironment: A driving force for breast cancer progression. Biochim Biophys Acta 1863: 382-391, 2016

11. Kroemer G and Pouyssegur J: Tumor cell metabolism: Cancer's Achilles' heel. Cancer Cell 13: 472-482, 2008.

12. Bernardi R and Gianni L: Hallmarks of triple negative breast cancer emerging at last? Cell Res 24: 904-905, 2014.

13. Cancer Genome Atlas N; Cancer Genome Atlas Network: Comprehensive molecular portraits of human breast tumours. Nature 490: 61-70, 2012.

14. Stopeck AT, Brown-Glaberman U, Wong HY, Park BH, Barnato SE, Gradishar WJ, Hudis CA and Rugo HS: The role of targeted therapy and biomarkers in breast cancer treatment. Clin Exp Metastasis 29: 807-819, 2012.

15. Wang GL, Jiang BH, Rue EA and Semenza GL: Hypoxia-inducible factor 1 is a basic-helix-loop-helix-PAS heterodimer regulated by cellular $\mathrm{O}_{2}$ tension. Proc Natl Acad Sci USA 92: 5510-5514, 1995

16. Semenza GL: Hypoxia-inducible factors: Mediators of cancer progression and targets for cancer therapy. Trends Pharmacol Sci 33: 207-214, 2012

17. Semenza GL: Hypoxia-inducible factor 1: Master regulator of $\mathrm{O}_{2}$ homeostasis. Curr Opin Genet Dev 8: 588-594, 1998.

18. Ke Q and Costa M: Hypoxia-inducible factor-1 (HIF-1). Mol Pharmacol 70: 1469-1480, 2006.

19. Smoliga JM, Baur JA and Hausenblas HA: Resveratrol and health - a comprehensive review of human clinical trials. Mol Nutr Food Res 55: 1129-1141, 2011.

20. Aggarwal BB, Bhardwaj A, Aggarwal RS, Seeram NP Shishodia $S$ and Takada Y: Role of resveratrol in prevention and therapy of cancer: Preclinical and clinical studies. Anticancer Res 24: 2783-2840, 2004

21. Jeong SH, Song IS, Kim HK, Lee SR, Song S, Suh H, Yoon YG, Yoo YH, Kim N, Rhee BD, et al: An analogue of resveratrol HS-1793 exhibits anticancer activity against MCF-7 cells via inhibition of mitochondrial biogenesis gene expression. Mol Cell 34: 357-365, 2012.

22. Kim HJ, Yang KM, Park YS, Choi YJ, Yun JH, Son CH, Suh HS, Jeong MH and Jo WS: The novel resveratrol analogue HS-1793 induces apoptosis via the mitochondrial pathway in murine breast cancer cells. Int J Oncol 41: 1628-1634, 2012.

23. Jeong SH, Jo WS, Song S, Suh H, Seol SY, Leem SH, Kwon TK and Yoo YH: A novel resveratrol derivative, HS1793, overcomes the resistance conferred by Bcl-2 in human leukemic U937 cells. Biochem Pharmacol 77: 1337-1347, 2009.

24. Jeong NY, Yoon YG, Rho JH, Lee JS, Lee SY, Yoo KS, Song S, Suh H, Choi YH and Yoo YH: The novel resveratrol analog HS-1793-induced polyploid LNCaP prostate cancer cells are vulnerable to downregulation of Bcl-xL. Int J Oncol 38 $1597-1604,2011$

25. Jeong SH, Lee JS, Jeong NY, Kim TH, Yoo KS, Song S, Suh H, Kwon TK, Park BS and Yoo YH: A novel resveratrol analogue HS-1793 treatment overcomes the resistance conferred by Bcl-2 and is associated with the formation of mature PML nuclear bodies in renal clear cell carcinoma Caki-1 cells. Int J Oncol 35: 1353-1360, 2009

26. Jeong MH, Yang KM, Choi YJ, Kim SD, Yoo YH, Seo SY, Lee SH, Ryu SR, Lee CM, Suh H, et al: Resveratrol analog, HS-1793 enhance anti-tumor immunity by reducing the $\mathrm{CD} 4^{+} \mathrm{CD} 25^{+}$regulatory T cells in FM3A tumor bearing mice. Int Immunopharmacol 14: 328-333, 2012.
27. Kim JA, Kim DH, Hossain MA, Kim MY, Sung B, Yoon JH, Suh H, Jeong TC, Chung HY and Kim ND: HS-1793, a resveratrol analogue, induces cell cycle arrest and apoptotic cell death in human breast cancer cells. Int J Oncol 44: 473-480, 2014

28. Um HJ, Bae JH, Park JW, Suh H, Jeong NY, Yoo YH and Kwon TK: Differential effects of resveratrol and novel resveratrol derivative, HS-1793, on endoplasmic reticulum stress-mediated apoptosis and Akt inactivation. Int J Oncol 36: 1007-1013, 2010.

29. Kim DH, Hossain MA, Kim MY, Kim JA, Yoon JH, Suh HS, Kim GY, Choi YH, Chung HY and Kim ND: A novel resveratrol analogue, HS-1793, inhibits hypoxia-induced HIF-1 $\alpha$ and VEGF expression, and migration in human prostate cancer cells. Int J Oncol 43: 1915-1924, 2013

30. Kim DH, Kim MJ, Sung B, Suh H, Jung JH, Chung HY and Kim ND: Resveratrol analogue, HS-1793, induces apoptotic cell death and cell cycle arrest through downregulation of AKT in human colon cancer cells. Oncol Rep 37: 281-288, 2017.

31. Tada H, Shiho O, Kuroshima K, Koyama M and Tsukamoto K: An improved colorimetric assay for interleukin 2. J Immunol Methods 93: 157-165, 1986.

32. Sung B, Park S, Ha YM, Kim DH, Park CH, Jung KJ, Kim MS, Kim YJ, Kim MK, Moon JO, et al: Salicylideneamino-2thiophenol modulates nuclear factor- $\kappa \mathrm{B}$ through redox regulation during the aging process. Geriatr Gerontol Int 15: 211-219, 2015.

33. Semenza GL: Hypoxia, clonal selection, and the role of HIF-1 in tumor progression. Crit Rev Biochem Mol Biol 35: 71-103, 2000.

34. Folkman J: Role of angiogenesis in tumor growth and metastasis. Semin Oncol 29 (Suppl 16): 15-18, 2002.

35. Schindl M, Schoppmann SF, Samonigg H, Hausmaninger $H$ Kwasny W, Gnant M, Jakesz R, Kubista E, Birner P and Oberhuber G; Austrian Breast and Colorectal Cancer Study Group: Overexpression of hypoxia-inducible factor 1alpha is associated with an unfavorable prognosis in lymph node-positive breast cancer. Clin Cancer Res 8: 1831-1837, 2002.

36. Huang M, Chen Q, Xiao J, Yao T, Bian L, Liu C and Lin Z: Overexpression of hypoxia-inducible factor- $1 \alpha$ is a predictor of poor prognosis in cervical cancer: A clinicopathologic study and a meta-analysis. Int J Gynecol Cancer 24: 1054-1064, 2014.

37. Hubbi ME, Gilkes DM, Hu H, Kshitiz, Ahmed I and Semenza GL: Cyclin-dependent kinases regulate lysosomal degradation of hypoxia-inducible factor $1 \alpha$ to promote cell-cycle progression. Proc Natl Acad Sci USA 111: E3325-E3334, 2014.

38. Forsythe JA, Jiang BH, Iyer NV, Agani F, Leung SW, Koos RD and Semenza GL: Activation of vascular endothelial growth factor gene transcription by hypoxia-inducible factor 1 . Mol Cell Biol 16: 4604-4613, 1996.

39. Carbó N, Costelli P, Baccino FM, López-Soriano FJ and Argilés JM: Resveratrol, a natural product present in wine, decreases tumour growth in a rat tumour model. Biochem Biophys Res Commun 254: 739-743, 1999.

40. Wu SL, Sun ZJ, Yu L, Meng KW, Qin XL and Pan CE: Effect of resveratrol and in combination with 5-FU on murine liver cancer. World J Gastroenterol 10: 3048-3052, 2004.

41. Kimura $\mathrm{Y}$ and Okuda $\mathrm{H}$ : Resveratrol isolated from Polygonum cuspidatum root prevents tumor growth and metastasis to lung and tumor-induced neovascularization in Lewis lung carcinoma-bearing mice. J Nutr 131: 1844-1849, 2001.

42. Marier JF, Vachon P, Gritsas A, Zhang J, Moreau JP and Ducharme MP: Metabolism and disposition of resveratrol in rats: Extent of absorption, glucuronidation, and enterohepatic recirculation evidenced by a linked-rat model. J Pharmacol Exp Ther 302: 369-373, 2002.

43. Garvin S, Ollinger K and Dabrosin C: Resveratrol induces apoptosis and inhibits angiogenesis in human breast cancer xenografts in vivo. Cancer Lett 231: 113-122, 2006.

44. Castillo-Pichardo L, Cubano LA and Dharmawardhane S: Dietary grape polyphenol resveratrol increases mammary tumor growth and metastasis in immunocompromised mice. BMC Complement Altern Med 13: 6, 2013

45. Bove K, Lincoln DW and Tsan MF: Effect of resveratrol on growth of 4T1 breast cancer cells in vitro and in vivo. Biochem Biophys Res Commun 291: 1001-1005, 2002.

46. Jeong SK, Yang K, Park YS, Choi YJ, Oh SJ, Lee CW, Lee KY, Jeong MH and Jo WS: Interferon gamma induced by resveratrol analog, HS-1793, reverses the properties of tumor associated macrophages. Int Immunopharmacol 22: 303-310, 2014. 